\title{
NANOTOXICOLOGIA DE NANOPARTÍCULAS DE PRATA: TOXICIDADE EM ANIMAIS E HUMANOS
}

\author{
Nelson Durán ${ }^{\mathrm{a}, *}$, Wallace R. Rolim ${ }^{\mathrm{b}}$, Marcela Durána, Wagner J. Fávaroa e Amedea B. Seabra ${ }^{\mathrm{b}}$ \\ anstituto de Biologia, Universidade Estadual de Campinas, 13083-862 Campinas - SP, Brasil \\ ${ }^{b}$ Centro de Ciências Naturais e Humanas, Universidade Federal do ABC, 09210-580 Santo André - SP, Brasil
}

Recebido em 17/07/2018; aceito em 05/11/2018; publicado na web em 17/12/2018

\begin{abstract}
NANOTOXICOLOGY OF SILVER NANOPARTICLES: TOXICITY IN ANINALS AND HUMANS. Silver nanoparticles (AgNPs) have important antimicrobial activity. These nanoparticles are present in several commercial products, leading to human and environmental exposure. This review presents a critical view of the toxic effects of Ag NPs on in vitro and in vivo biological models. It is noteworthy that the toxicity of AgNPs is dependent on several parameters, such as the preparation mode of the colloidal suspension of the nanoparticles, their state of aggregation, the chemical nature of the nanoparticles, surface size, morphology, dose, cell type, nature of the living organism, among others. In general, low concentrations of AgNPs do not present significant toxicity in in vitro and in vivo assays, however with the increase of the concentration of AgNPs there is a significant increase of nanoparticle toxicity and its accumulation in diverse tissues/organs. It should be emphasized that in living organisms, exposure routes should be taken into account, such as ingestion and inhalation. At high concentrations, inhalation or ingestion of AgNPs can cause adverse effects and even lead to human death. In this context, the importance of further studies of the toxicity of AgNPs in several in vitro and in vivo models is emphasized.
\end{abstract}

Keywords: silver nanoparticles; nanotoxicity; in vitro toxicity; in vivo toxicity.

\section{INTRODUÇÃO}

A nanotoxicologia é um ramo da toxicologia dedicado ao estudo dos efeitos toxicológicos de nanomateriais em diferentes sistemas biológicos, incluindo células, tecidos e organismos vivos. ${ }^{1}$ Uma década de pesquisa envolvendo a nanotoxicologia tem mostrado que as interações entre os nanomateriais com células, animais, seres humanos e meio ambiente são extremamente complexas. ${ }^{2}$ Atualmente, os pesquisadores ainda estão tentando entender, em detalhes, como as propriedades físico-químicas e morfológicas dos nanomateriais podem influenciar essas interações, e assim determinar o impacto final dos nanomateriais na saúde e no meio ambiente. ${ }^{3}$ Toxinas são compostos químicos naturais ou sintéticos capazes de causar efeitos adversos ou danos (toxicidade) nos sistemas biológicos. Entretanto, esses efeitos são dependentes da dose. Em geral, a dose é definida como a massa de um composto químico por unidade de peso corpo$\mathrm{ral}$ (g/kg de peso corporal). ${ }^{4}$ As propriedades morfológicas e físico-química dos nanomateriais causam grande impacto na interação do nanomaterial com as células, em meio biológico, e dessa forma impactam sua toxicidade. Entretanto, apesar da nanotoxicologia estudar os efeitos tóxicos de nanomateriais, os efeitos de tamanho dos materiais são importantes ao compará-los com materiais em escala microscópica ou macroscópica. Logo, o conceito de dose para nanotoxicologia se baseia em diferentes parâmetros, como: tamanho da partícula e sua área superficial, morfologia, composição, química da superfície, estado de aglomeração/agregação, etc. ${ }^{3,5}$ De fato, todos estes parâmetros influenciam criticamente na determinação da dose das nanopartículas e, consequentemente, na avaliação precisa da sua toxicidade. Singh et al. ${ }^{4}$ resumiram esses conceitos na emergente área da nanotoxicologia. Contudo, deve-se ter em mente que humanos são animais, portanto, proteção contra a toxicidade de agentes externos e determinação de valores máximos de exposição seriam impossíveis de serem determinados sem a possibilidade de estudar seus efeitos sobre animais de laboratório.
Existem já algumas estratégias para estudos de interação de nanopartículas e sistemas biológicos. ${ }^{6}$

Nas próximas sessões desse trabalho, serão demonstrados os conceitos aplicados à nanotoxicologia considerando algumas das nanopartículas mais estudadas nos últimos anos e utilizadas comercialmente: as nanopartículas de prata (NPs de Ag).

\section{O que são nanopartículasde prata (NPs de Ag)?}

Existem várias definições segundo as agências envolvidas com nanotecnologia e nanopartículas. A recomendação atual (desde 2016) para definir um nanomaterial, de acordo com a Comunidade Européia é: "um material natural, incidental ou manufaturado contendo partículas, em estado não agregado ou agregado, no qual 50\% ou mais das partículas na distribuição de tamanho numérico, uma ou mais dimensões externas estiverem na faixa de tamanho $1 \mathrm{~nm}$ 100 nm" (http://ec.europa.eu/environment/chemicals/nanotech/faq/ definition_en.htm).

As NPs de Ag são um dos nanomateriais mais estudados atualmente. São as nanopartículas mais utilizadas do ponto de vista industrial e comercial graças às suas propriedades características, como estabilidade química, maleabilidade, flexibilidade, elevada condutividade elétrica e térmica, atividade catalítica, relativo baixo custo de produção e principalmente potente ação antimicrobiana frente a bactérias, vírus, fungos e protozoários. Dessa forma, as NPs de Ag são amplamente utilizadas em várias aplicações como agentes antibacterianos, antifúngicos, antivirais, anti-inflamatórios, antitumorais, regeneradores, biossensores e catalisadores. Elas estão presentes em produtos na indústria de alimentos, têxtil, perfumaria, farmacêutica, agrícola, higiene, produtos de limpeza, tintas, eletroeletrônicos, entre outras. ${ }^{7-17}$ Possuem uma ampla aplicabilidade, como por exemplo, em medicina, cosmetologia, no recobrimento de dispositivos médicos, na confecção de nanogéis, nanoloções, na preparação de materiais para recobrimento de lesões cutâneas. ${ }^{11-14}$ 


\section{Importância das NPs de Ag na pesquisa e no mercado}

Ao se realizar uma busca em plataformas de busca de artigos científicos, como o Web of Science - ISI, pode-se encontrar numerosas citações em função de vários parâmetros. A Figura 1A mostra as referências obtidas utilizando-se como palavras chaves na busca: "silver nanoparticles" e "toxicology" (6962 citações). A Figura 1B mostra que usando as palavras "AgNPs" e "nanotoxicology" na busca, aparece um valor de 9359 citações.

A Figura 2 apresenta uma tendência do Mercado Global baseado uso de NPs de Ag em aplicações eletroeletrônicas, na área da saúde, das indústrias de alimentos, bebidas e têxtil. O mercado global de NPs de Ag foi analisado desde 2012 com perspectivas até 2022, destacando a utilização em toneladas dessas nanopartículas. (Publicado: Maio 2015, ISBN Code: 978-1-68038-413-0 (http://www.grandviewresearch.com/industry-analysis/silver-nanoparticles-market) (Figura 2). Uma análise importante foi realizada pela "Scientific Committee on Emerging and Newly Identified Health Risks" (SCENIHR) mostrando que as NPs de Ag são utilizadas em aproximadamente 243 produtos comerciais. $^{18}$

Em vista do numeroso uso das AgNPs (Figura 2), a Agência de Proteção Ambiental dos EUA (EPA), recentemente, começou a exigir que os fabricantes de NPs de Ag registrem seus produtos, indicando que as nanopartículas podem trazer riscos diferentes do que a prata regular (não nanométrica). Entretanto, existem alguns questionamentos a respeito deste ponto. Os clientes, por sua vez, muitas vezes não podem verificar se os produtos contêm prata de tamanho nanométrico, já que não há obrigação de se informar no rótulo do produto a presença de nanomaterais. A França foi um dos primeiros países a exigir este tipo de notificação nas bulas de cosméticos. ${ }^{5}$ A Figura $3 \mathrm{~A}$ mostra o aumento do número de produtos comerciais com nanomateriais no período de 2005-2013, enquanto que a Figura 3B mostra a porcentagem de produtos associados a um específico material no ano de 2013.

É possível observar que no ano de 2013 mais de 50\% dos nanomateriais produzidos foram baseados em NPs de Ag. Outras predições baseadas nos conhecimentos do mercado relatam que a comercialização de produtos contendo NPs de Ag poderia chegar a movimentar 1.9 bilhões de dólares em 2020. Isto significaria uma expansão a uma velocidade de crescimento anual (Compound Annual Growth Rate-CAGR) de $15.4 \%$ entre 2014 e 2020. (http://www. transparencymarketresearch.-com/pressrelease/global-nanosilver-market.htm). Assim, as informações e projeções na produção e aplicação de NPs de Ag são enormes, e isso indica o quão importante
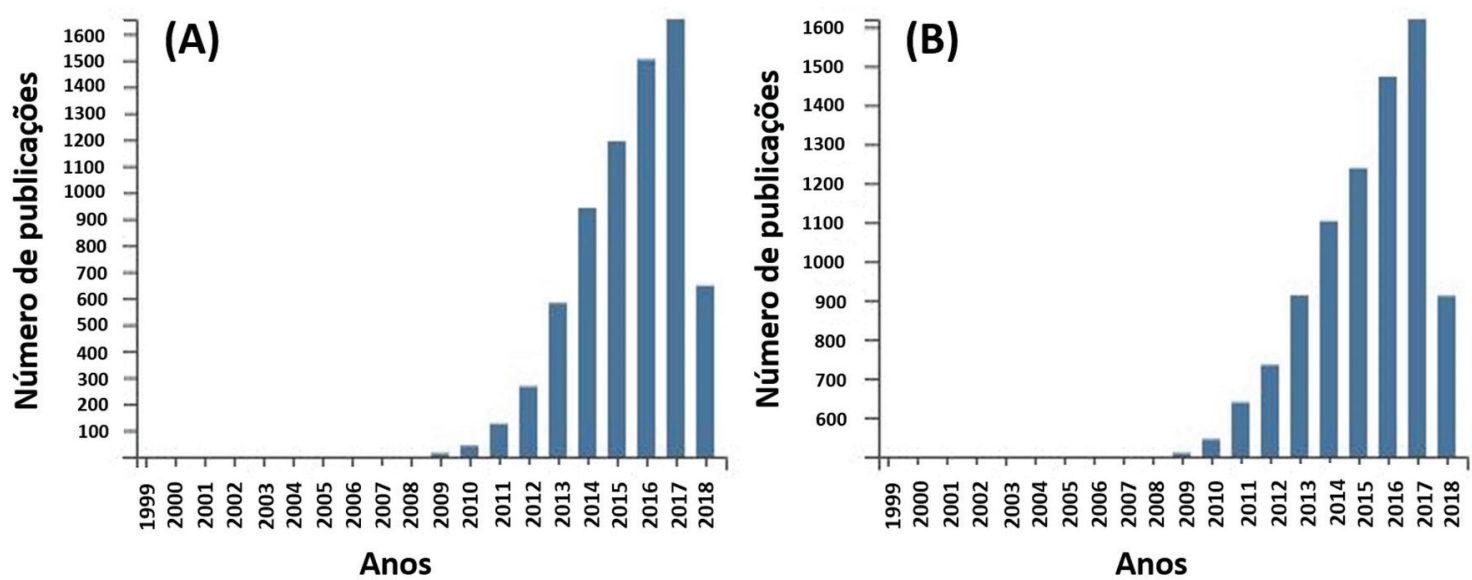

Figura 1. Resultados da busca de trabalhos na plataforma Web of Science, usando como palavras chaves "silver nanoparticles" e "toxicology" (A) e resultados da busca de trabalhos usando como palavras chaves "AgNPs" e "nanotoxicology" (B). Data da busca: 12 junho de 2018

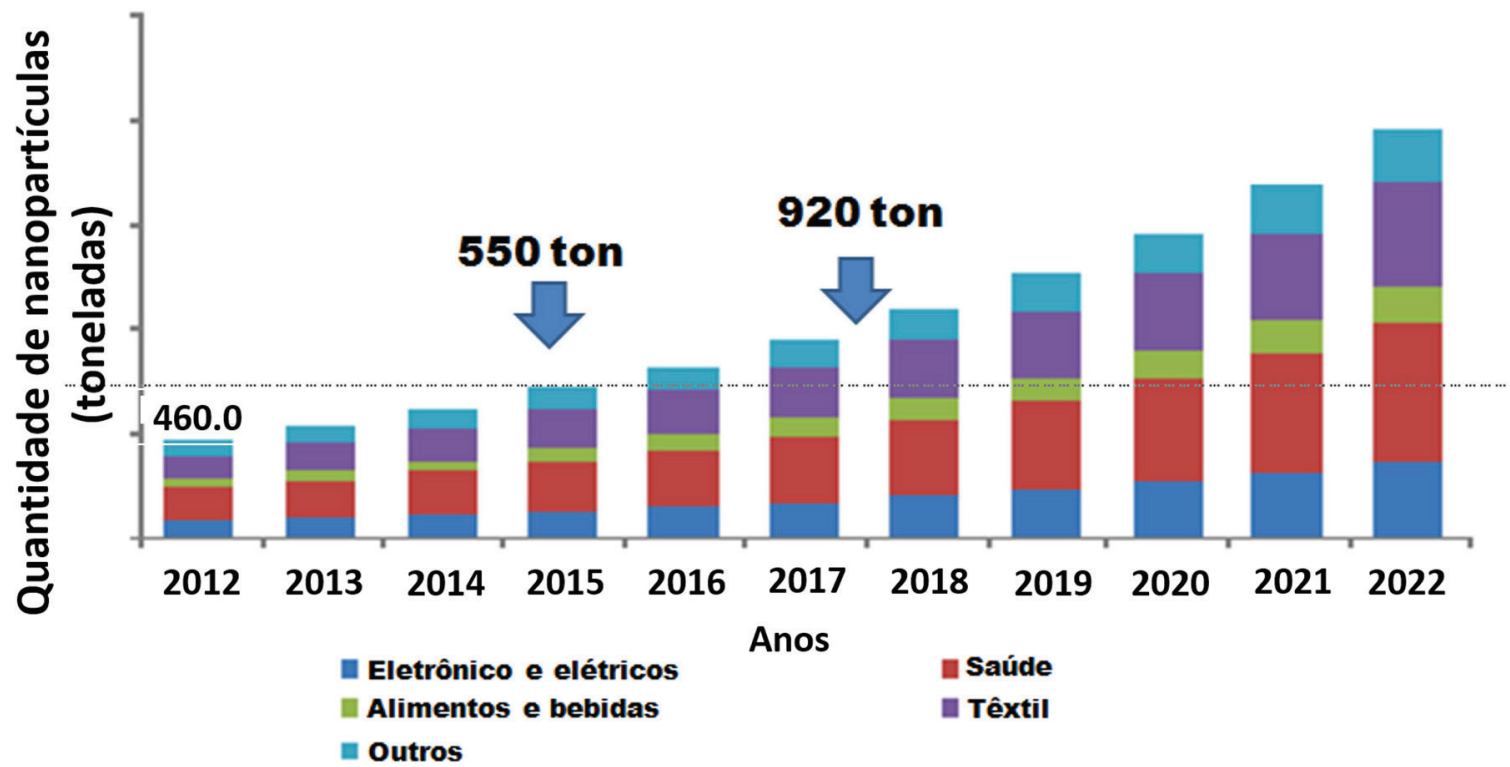

Figura 2. Mercado e produção em toneladas de NPs de Ag. (Fonte: https://www.grandviewresearch.com/industry-analysis/silver-nanoparticles-market, acessado em 24 de maio de 2018) 

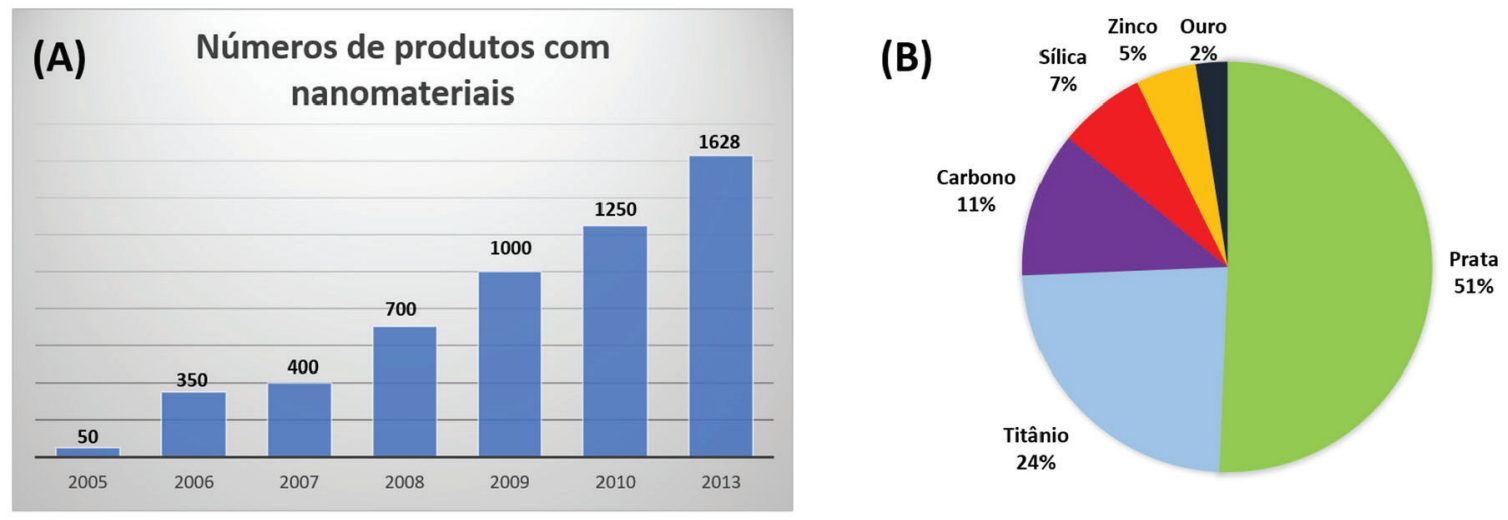

Figura 3. Mercado e produção em toneladas de NPs de Ag. (A): o aumento do número de produtos comerciais com nanomateriais no período de 2005 -2013. (B): Porcentagem de elementos químicos associados a produtos comerciais no ano de 2013. http://ec.europa.eu/health/scientific_committees/consultations/ public_consultations/scenihr_consultation_17_en.htm. (Fonte: The Project on Emerging Nanotechnologies) ${ }^{18}$

são os aspectos de segurança e avaliação dos seus riscos para saúde humana e ambiental. ${ }^{18-23}$

\section{ROTAS DE SÍNTESE DE NPs DE Ag}

Embora existam diversificados métodos de produção de NPs de Ag, duas rotas são as mais utilizadas: a síntese por redução química (tradicionalmente empregada) e a síntese biogênica ou biológica, desenvolvida mais recentemente devido a sua rapidez, simplicidade e sustentabilidade. ${ }^{21}$ Ambas as rotas são baseadas na redução de íons prata $\left(\mathrm{Ag}^{+}\right)$a $\mathrm{Ag}^{0}$, formando as NPs de $\mathrm{Ag}$.

Na rota química de preparação de NPs de Ag, os agentes redutores de $\mathrm{Ag}^{+}$mais utilizados são o citrato de sódio e borohidreto de sódio (Figura 4A). ${ }^{22}$ Além disso, a síntese química também apresenta versatilidade e outros agentes redutores utilizados são o poli (álcoolvinílico) (PVA) e poli (etilenoglicol) (PEG), polímeros biocompatíveis que também atuam como agente estabilizantes. ${ }^{11,23}$ Em geral, a síntese química forma NPs de Ag com considerável reprodutibilidade, permitindo o controle do tamanho e dispersão das nanopartículas, controlando os parâmetros experimentais. Destaca-se que a síntese química permite o maior controle do tamanho das NPs obtidas. As sínteses químicas apresentam versatilidade e algumas rotas podem empregar meio aquoso, ausência de reagentes tóxicos e condições experimentais brandas. Entretanto, em algumas rotas sintéticas químicas empregam-se reagentes tóxicos, podendo gerar sub-produtos tóxicos, alguns protocolos requerem a presença de atmosfera inerte ou controle da temperatura. Além disso, a após a síntese das NPs de Ag pela rota química, a superfície das mesmas deve ser recoberta a fim de evitar a oxidação as nanopartículas e sua aglomeração.

Alternativamente, a rota biogênica vem ganhando considerável importância nos últimos anos (Figura 4B). ${ }^{24-27}$ Trata-se de uma opção economicamente e ecologicamente viável para a produção de nanopartículas metálicas, incluindo as NPs de Ag. A síntese biogênica é considerada simples, de baixo custo, sustentável e pode ser realizada sob temperatura e pressão ambientes, sem uso de agentes estabilizantes externos. ${ }^{14,28,29}$ As desvantagens da síntese biogênica podem ser elencadas como a maior dificuldade de escalonamento e rendimento das nanopartículas e, portanto, mais estudos são necessários para o seu desenvolvimento. A síntese biogênica é realizada por extratos vegetais, fungos, bactérias, algas e leveduras. ${ }^{30}$ Destaca-se que na síntese biogênica o agente redutor também é responsável pelo revestimento da superfície da nanopartículas obtidas, aumentando sua estabilidade e evitando sua aglomeração.

A Figura 4 representa esquematicamente a síntese química (A) e biogênica (B) de NPs de Ag. Na síntese química, a redução dos íons
$\mathrm{Ag}^{+}$foi realizada pelo borohidreto de sódio $\left(\mathrm{NaBH}_{4}\right)$. Na Figura $4 \mathrm{~B}$ a redução dos íons $\mathrm{Ag}^{+}$ocorre a partir de redutases presentes no extrato do fungo Fusarium oxysporum e transferência de elétron. Ressaltase que na síntese biogênica de nanopartículas, os agentes biológicos responsáveis pela redução dos íons agem também como agentes pacificadores das nanopartículas metálicas obtidas, aumentando a estabilidade da suspensão coloidal de nanopartículas, evitando a oxidação e aglomeração das partículas. Além disso, a presença desses agentes biológicos na superfície das nanopartículas obtidas também pode oferecer alguma funcionalidade à nanopartícula. ${ }^{31,32}$ Em relação à síntese de NPs de Ag, recentemente vários trabalhos importantes foram publicados. ${ }^{8,33-38}$ Contudo, é importante destacar que as NPs de $\mathrm{Ag}$ produzidas por diferentes métodos apresentaram diferentes características físico-químicas e morfológicas e, portanto, diferentes interações no meio biológico resultando em diferentes toxicidades.

\section{PARÂMETROS IMPORTANTES QUE INFLUENCIAM A TOXICIDADE DAS NPS DE Ag}

Cada nanopartícula (incluindo-se outras nanopartículas além das NPs de Ag) é única, em função da sua rota de preparação, tamanho, estado de agregação, estabilidade em meio biológico, natureza química do revestimento, carga superficial, etc. Destaca-se que tal variabilidade no processo de síntese de nanopartículas, incluindo as NPs de Ag, poderia ser um problema para o uso comercial das mesmas. O controle nos parâmetros de síntese de NPs de Ag é necessário para garantir a reprodutibilidade de sua produção. Nesse sentido, nesta seção serão abordados alguns fatores importantes que influenciam a toxicidade das NPs de Ag em células de humanos e animais.

\section{Preparação da suspensão}

Park et al. ${ }^{39}$ avaliaram a toxicidade de uma suspensão comercial de NPs de Ag recobertas com citrato de sódio, preparada a partir de diferentes métodos. Os resultados mostram que a suspensão fracionada (agitada e sedimentada) foi menos tóxica para o organismo aquático modelo Daphnia $\left(13,8 \mu \mathrm{g} \mathrm{L}^{-1}\right)$, em comparação com a suspensão coloidal $\left(6.1 \mu \mathrm{g} \mathrm{L}^{-1}\right)$ sem tratamento algum, sugerindo que o método de preparação da suspensão de NPs de Ag foi um fator crítico na determinação da toxicidade das nanopartículas (Tabela 1).

Entretanto, as diferenças de toxicidade causadas pela suspensão de NPs de Ag não foram observadas em peixe (Oryzias latipes). Análises de microscopia eletrônica de transmissão (TEM) mostraram que no caso da Daphnia, a suspensão coloidal é mais tóxica que a fracionada. Esse resultado pode ser explicado devido à maior 


\section{SÍNTESE QUÍMICA PELA REAÇÃO COM BOROHIDRETO DE SÓDIO}

\section{SÍNTESE BIOGÊNICA}

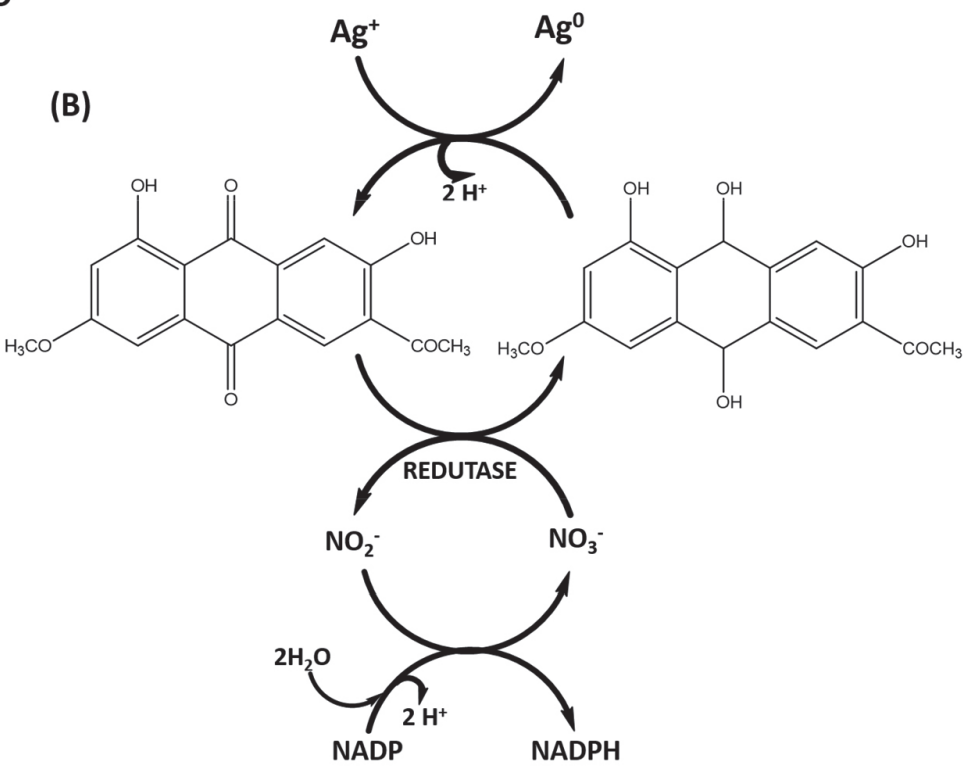

Figura 4. Síntese química (A) e biogênica de NPs de Ag (B) (modificado a partir das referências 22, com permissão da American Chemical Society, e 26, artigo de acesso livre distribuído sob os termos da Creative Commons Attribution License: http://creativecommons.org/licenses/by/2.0)

Tabela 1. Parâmetros de toxicidades de suspensão de NPs de Ag comercial, preparada por diferentes métodos, em Daphnia magna e Oryzias latipes

\begin{tabular}{ccc}
\hline Tipo da amostra & $\begin{array}{c}\text { Daphnia magna } \\
\text { Total Ag EC } \\
(\mathrm{CI} 95 \%, \mu \mathrm{g} / \mathrm{L})\end{array}$ & $\begin{array}{c}\text { Oryzias latipes } \\
\text { Total Ag LC } \\
(\mathrm{CI} 95 \%, \mu \mathrm{g} / \mathrm{L})\end{array}$ \\
\hline AgNPs fracionada & $13,8 \mu \mathrm{g} / \mathrm{L}(13,3-14,0)$ & $64,7 \mu \mathrm{g} / \mathrm{L}\left(^{\mathrm{a}}\right)$ \\
AgNPs coloidal & $6,1 \mu \mathrm{g} / \mathrm{L}(5,6-6,5)$ & $76,0 \mu \mathrm{g} / \mathrm{L}(55,0-99,0)$ \\
$\mathrm{AgNO}_{3}$ & $2,1 \mu \mathrm{g} / \mathrm{L}(2,1-2,1)$ & $49,3 \mu \mathrm{g} / \mathrm{L}(43,9-55,3)$ \\
\hline
\end{tabular}

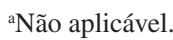

agregação que se observa na suspensão coloidal em relação à suspensão fracionada. No caso da Daphinia, a toxicidade das suspensões de NPs de Ag foi menor em comparação à toxicidade dos íons $\mathrm{Ag}^{+}$ (nitrato de prata $\left(\mathrm{AgNO}_{3}\right)$. As NPs de $\mathrm{Ag}$ possam liberar íons $\mathrm{Ag}^{+}$, o que geraria toxicidade. Os resultados apresentados na Tabela 1 mostram que o método utilizado para preparar a suspensão de AgNPs pode afetar a toxicidade das nanopartículas. Além disso, a Tabela 1 também mostra que as mesmas suspensões de nanopartículas, preparadas pelos métodos iguais, podem causar diferentes toxicidades em diferentes organismos. ${ }^{39}$

Ressalta-se que o impacto do envelhecimento das suspensões coloidais de nanopartículas na toxicidade das mesmas ainda é pouco investigado devido à falta de metodologias adequadas. Entretanto, recentemente, um estudo demonstrou este efeito na toxicidade aguda de nanopartículas em matrizes reais e complexas, que provavelmente está associado à dissolução da amostra. ${ }^{40}$

\section{Química de superfície das nanopartículas}

Considerando que a interação de NPs de Ag com o meio biológico, por exemplo, uma bactéria, ocorre por interação direta da nanopartículas com a entidade biológica, a química de superfície do nanomaterial é de grande importância para a ação biológica da nanopartícula. Dessa forma, a natureza química do revestimento da superfície da nanopartículas terá uma interação direta com a bactéria, ou outro microorganismo, desempenhando um papel importante na toxicidade da NP de Ag. Nesse sentido, estudos mostram como o revestimento da superfície de NPs de Ag pode atuar de forma diferente na toxicidade de NPs de Ag. ${ }^{16,36}$ A natureza química do revestimento da superfície de NPs de Ag pode impactar significativamente sua toxicidade. A fim de avaliar esse parâmetro, foi realizado um estudo de exposição oral por 28 dias em ratos expostos a NPs de Ag (tamanho $<20 \mathrm{~nm}$ ), NPs de Ag recobertas com polivinil pirrolidona (PVP) (NPs de Ag-PVP) (tamanho < 15) ([Ag]= $90 \mathrm{mg} \mathrm{kg}^{-1}$ peso do rato) e $\mathrm{AgNO}_{3}\left([\mathrm{Ag}]=9 \mathrm{mg} \mathrm{kg}^{-1}\right.$ de peso do rato), em uma solução única. ${ }^{41}$ Após dissecação dos animais foi encontrada prata em todos os órgãos, destacando-se altas quantidades no fígado e baço. A concentração da prata nos órgãos correlacionou-se com a quantidade de $\mathrm{Ag}^{+}$na suspensão de NPs de $\mathrm{Ag}$, indicando que principalmente $\mathrm{Ag}^{+}$, e uma menor extensão NPs de Ag, passou ao intestino do rato exposto. Em todos os grupos estudados, a prata foi removida da maioria dos órgãos após 8 semanas após a dosagem. Entretanto, após esse período, ainda houve a presença de prata no cérebro e dos testículos dos animais, em todos os grupos tratados. Todos os biomarcadores não relevaram hepatotoxicidade ou imunotoxicidade devido à exposição à prata. Portanto, a exposição oral dos animais a NPs de Ag parece ser muito similar à exposição com íons $\mathrm{Ag}^{+}$. Entretanto, a longa permanência da prata no cérebro e testículos dos animais deve ser considerada na avaliação de potencial risco das NPs de Ag. ${ }^{41}$

NPs de Ag são responsáveis por diminuir as atividades das enzimas antioxidantes e desequilibrar o estado oxidativo nas células, entretanto, a funcionalização da superfície dessas nanopartículas com albumina do soro bovino (BSA) e polietileno glicol (PEG) ajuda a proteger o efeito adverso de NPs de Ag nas células, indicando que a modificação da superfície das nanopartículas é fator importante na sua toxicidade ${ }^{42}$ Ressalta-se que outro aspecto relevante na modificação da superfície das NPs de Ag em meio biológico são as proteínas corona, que podem mudar completamente sua a atividade e toxicidade das nanopartículas. ${ }^{43}$

\section{Tamanho da nanopartícula}

A toxicidade de nanopartículas pode ser afetada pelo tamanho de nanopartículas. Por exemplo, de maneira geral, reporta-se que 
nanopartículas menores apresentam uma tendência a maior habilidade de penetração celular e consequentemente, efeitos de toxicidade. Apesar de haver exceções, nanopartículas menores tendem a ser mais tóxicas em função da maior área superficial, promovendo uma maior área de contato e interação entre a nanopartículas e a entidade biológica, como por exemplo, uma bactéria. Ressalta-se que o tamanho da nanopartícula não é o único fator que caracteriza a toxicidade da nanopartícula. ${ }^{16,36} \mathrm{~A}$ toxicidade em função do tamanho das NPs de Ag foi avaliada em camundongos tratados com diferentes tamanhos de nanopartículas, numa dose única de $1 \mathrm{mg} \mathrm{kg}^{-1}$ por 14 dias por administração oral. ${ }^{39}$ As NPs de Ag de menores tamanhos que foram estudadas (22-71 nm) foram encontradas em diferentes órgãos incluindo cérebro, pulmão, fígado, rins e testículos, entretanto, as nanopartículas de tamanho maiores ( $323 \mathrm{~nm}$ ) não foram detectadas nos tecidos. Os níveis de marcadores bioquímicos aumentaram significativamente no soro dos animais tratados com as nanopartículas menores, entretanto, o mesmo não foi observado para as nanopartículas maiores. Em experimentos usando doses repetidas de NPs de Ag (tamanho médio de 42 $\mathrm{nm}$ ), durante 28 dias, em diferentes doses, foram observados efeitos adversos sobre o fígado e rins somente na dose alta $\left(1,00 \mathrm{mg} \mathrm{kg}^{-1}\right)$. Baseado nestes resultados, sugere-se que a administração oral repetida de NPs de Ag pode causar hepatotoxicidade e resposta inflamatória em camundongos. Além disso, nanopartículas menores tendem a ser mais tóxicas e a se concentrar em diversos órgãos/tecidos.

Liu e coautores avaliaram os efeitos citotóxicos de NPs de Ag comercias de diferentes tamanhos ( $5 \mathrm{~nm}, 20 \mathrm{~nm}$ e $50 \mathrm{~nm}$ ) recobertas com PVP frente a diferentes linhagens de células humanas, a saber: A549, SGC-7901, HepG2 e MCF-7. Os autores observaram maiores efeitos citotóxicos com as nanopartículas menores, ou seja, de $5 \mathrm{~nm}$, em comparação com as nanopartículas maiores $(20 \mathrm{~nm}$ e $50 \mathrm{~nm})$. Além disso, houve uma maior internalização das nanopartículas menores.
Esses resultados indicam a relação entre tamanho e toxicidade das nanopartículas, para nanopartículas com um mesmo revestimento. ${ }^{44}$

No entanto, recentemente, um estudo usando como modelo o mexilhão Mytilus galloprovincialis destacou que não só o tamanho da nanopartícula é importante para impactar sua toxicidade, como também os diferentes mecanismos de internalização das mesmas poderiam ser fatores-chaves que afetam o potencial do nanomaterial no meio para induzir a imunomodulação. ${ }^{45}$

\section{Morfologia da nanopartícula}

Tak et al. sintetizaram NPs de Ag com diferentes morfologias, a partir de uma solução de borohidreto de sódio (agente redutor) na presença de citrato de sódio, como agente estabilizador. ${ }^{46}$ Os autores obtiveram nanopartículas com morfologias triangulares, esféricas e de bastões (Figura 5). As nanopartículas em forma de bastões apresentaram um tamanho de $50 \mathrm{~nm}$ de comprimento e $20 \mathrm{~nm}$ de diâmetro, enquanto que as nanopartículas esféricas apresentaram um tamanho médio de $50 \mathrm{~nm}$ e as nanopartículas triangulares apresentaram uma espessura equilateral de $2 \mathrm{~nm}$ e um comprimento de $2 \mathrm{~nm}$. Foram realizados ensaios in vivo em ratos SKH-1. Após 5 dias, foi retirado sangue do coração do animal, que foi analisado por ICP-MS para quantificação de prata. Observou-se que houve uma maior permeação de NPs de Ag com na forma de varas $\left(105,57 \pm 5,43 \mathrm{ng} \mathrm{mL}^{-1}\right)$, comparados com uma permeação de $50 \pm 2,50 \mathrm{ng} \mathrm{mL}^{-1}$ e 39,29 $\pm 1,96 \mathrm{ng}$ $\mathrm{mL}^{-1}$ de nanopartículas esféricas e triangulares, respectivamente. Os autores explicam que o tempo de exposição (5 dias) pode ter fornecido tempo suficiente para que as NPs de Ag penetrem lentamente o stratum corneum, epiderme e as camadas da derme. ${ }^{46}$ Além disso, a interação das NPs de Ag com as bactérias é dependente da morfologia das NPs. De fato, NPs de Ag na forma triangular, as quais apresentam
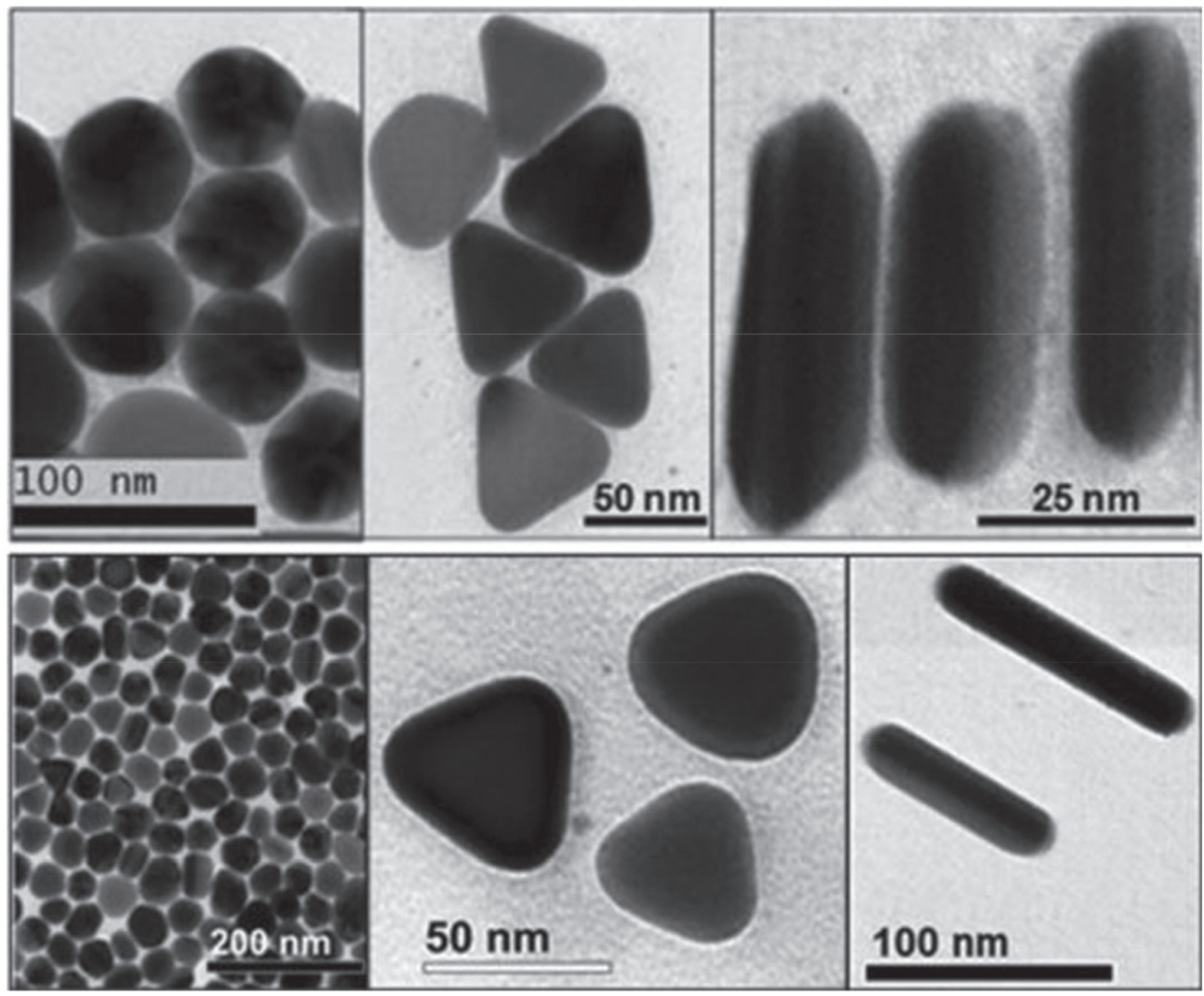

Figura 5. Imagens de microscopia eletrônica de transmissão de NPs de Ag nas formas esféricas, triangulares e de bastonetes, obtidas por diferentes protocolos sintéticos, (modificada a partir da referência 46, artigo de acesso livre distribuído sob os termos da Creative Commons Attribution 4.0 International License: http://creativecommons.org/licenses/by/4.0) 
um plano de rede (111) como o plano basal exibiu a ação biocida mais significativa, em comparação com nanopartículas esféricas e em forma de bastões, as quais apresentam predominantemente um plano de rede (100). ${ }^{46}$

\section{Efeito da dose}

No estudo de toxicidade oral de NPs de Ag (tamanho de $56 \mathrm{~nm}$ ) em ratos (F344) por 90 dias de exposição (0-500 $\left.\mathrm{mg} \mathrm{kg}^{-1}\right)$, observou-se uma diminuição significativa no peso corporal dos ratos machos, sem mudanças no consumo de alimentos ou água durante o período de exposição. A conclusão dos autores é que o órgão alvo das NPs de $\mathrm{Ag}$ foi o fígado, tanto nos ratos machos como nas fêmeas. O índice não observável de efeito adverso (do inglês "no observable adverse effect level") foi de $30 \mathrm{mg} \mathrm{kg}^{-1}$ e o menor nível observável de efeito adverso (do inglês "lowest observable adverse effect level") foi de $125 \mathrm{mg} \mathrm{kg}^{-1} \cdot{ }^{47}$

Pinzaru e coautores sintetizaram quimicamente NPs de Ag utilizando citrato de sódio e dodecil sulfato de sódio como agentes estabilizantes. Em seguida, as nanopartículas foram recobertas com PEG. Os efeitos tóxicos das nanopartículas de Ag com e sem o revestimento de PEG foram avaliadas in vitro frente a cultura de células de queratinócitos humanos (HaCat) e in vivo em testes não invasivos em camundongos sem pêlo SKH-1 com peso entre $30-35 \mathrm{~g}$. Foi administrada uma dose de $10 \mathrm{mg} \mathrm{kg}^{-1}$ de nanopartículas, diariamente durante 6 dias. Os resultados dos testes in vitro mostraram que em concentrações entre 0,1 e $3,0 \mu \mathrm{mol} \mathrm{L}{ }^{-1}$ o número de células viáveis não foi afetada após a adição da suspensão de nanopartículas durante diferentes períodos (24, 48 e $72 \mathrm{~h}$ ). As concentrações de 10 e $50 \mu \mathrm{mol}$ $\mathrm{L}^{-1}$ mostraram um efeito citotóxico induzido pelas nanopartículas, onde no tempo de 48 e $72 \mathrm{~h}$ o efeito foi mais significativo. Nos testes in vivo, foi avaliado o peso corpóreo dos camundongos. Foi possível observar que a via de injeção intraperitoneal não induziu sintomas nocivos aos camundongos na dose testada de $10 \mathrm{mg} \mathrm{kg}^{-1} .^{48}$

\section{Efeito dos íons $\mathrm{Ag}^{+}$}

As NPs de $\mathrm{Ag}$ podem liberar íons $\mathrm{Ag}^{+}$, quando são expostas a água e oxigênio, situação encontrada no meio biológico. A toxicidade das NPs de Ag está relacionada com a sua área superficial, uma maior área superficial pode promover uma maior liberação de íons $\mathrm{Ag}^{+}$, os quais estão relacionados à toxicidade e ação antimicrobiana das nanopartículas. Discute-se que parte considerável da toxicidade de NPs de $\mathrm{Ag}$ se deva à formação de íons $\mathrm{Ag}^{+}$no meio biológico. Assume-se que NPs de Ag podem ser internalizadas, e no interior das células liberar íons Ag+ causando estresse oxidativo, o qual pode promover a morte celular. ${ }^{16,36}$ Ressalta-se que o mecanismo exato de toxicidade as NPs de Ag ainda é tema de debate. ${ }^{36}$

\section{LIMITES CRÍTICOS DE EXPOSIÇÃO}

Os limites de exposição de componentes de prata nos Estados Unidos mostraram valores variados dependendo da fonte de informação, como mostrado na Tabela 2. Entretanto, no Brasil, a legislação estabelece nas águas potáveis um limite de $0,010 \mathrm{mg} \mathrm{L}^{-1}$ ou $10 \mu \mathrm{g} \mathrm{L}^{-1}$ de (Ag total). ${ }^{49}$

\section{Alguns exemplos de exposição e doses internas de nanomateriais a base de prata}

- Em 1979, 30 indivíduos foram expostos a nitrato de prata e óxido de prata. Seis apresentaram argiria e 20 tiveram argirose (deposição de prata nos olhos). As análises dos níveis de prata no
Tabela 2. Limites críticos de exposição para derivados de prata

\begin{tabular}{cc}
\hline Instituição & $\begin{array}{c}\text { Limite crítico de exposição para } \\
\text { derivados de prata }\end{array}$ \\
\hline $\begin{array}{c}\text { Conferência Americana } \\
\text { de Higienistas Industriais } \\
\text { Governamentais. }\end{array}$ & $\begin{array}{c}0,10 \mathrm{mg} / \mathrm{m}^{3} \text { ou } 0,1 \mu \mathrm{g} / \mathrm{L} \text { ou } 0,1 \mu \mathrm{g} / \mathrm{kg} \\
\text { de prata metálica }\end{array}$ \\
$0,10 \mathrm{mg} / \mathrm{m}^{3}$ ou $0,1 \mu \mathrm{g} / \mathrm{L}$ ou $0,1 \mu \mathrm{g} / \mathrm{kg}$ \\
de prata solúvel
\end{tabular}

Limite de exposição admissível

pel de administração de saúde de segurança ocupacional

Administração de segurança e saúde em minas

$0,01 \mathrm{mg} / \mathrm{m}^{3}$ ou $0,01 \mu \mathrm{g} / \mathrm{L}$ ou $0,01 \mu \mathrm{g} / \mathrm{kg}$ para qualquer forma de prata

Instituto Nacional de Segurança e Saúde Ocupacional ${ }^{37}$

Agência de Proteção Ambiental

(EPA) recomendação de concentração $50 \mathrm{ppb}$ ou $(50 \mu \mathrm{g} / \mathrm{L}$ ou $50 \mu \mathrm{g} / \mathrm{kg})$ de prata em água potável

$\mathrm{Na}$ água potável nos Estados Unidos tem-se encontrado níveis de prata sobre $80 \mathrm{ppb}(80 \mu \mathrm{g} / \mathrm{L}$ ou $80 \mu \mathrm{g} / \mathrm{kg})$. O consumo diário de prata numa dieta típica (US) chega a $27-88 \mu \mathrm{g}$ por dia ou $5-18 \mu \mathrm{g} / \mathrm{L} / \mathrm{dia}$ ou $5-18 \mu \mathrm{g} / \mathrm{kg} / \mathrm{dia}$. Nota: $1 \mathrm{mg} / \mathrm{m}^{3}=0,001 \mathrm{ppm}=0,001 \mathrm{mg} / \mathrm{kg}$

sangue apoiaram a natureza benigna da argíria $\left(18-26 \mu \mathrm{g} \mathrm{L}^{-1}\right) .{ }^{50}$

- Em 2006 uma pessoa com queimaduras utilizou um curativo chamado Acticoat ${ }^{\mathrm{R}}$ (contendo NPs de Ag com tamanho de $15 \mathrm{~nm}$ ) por 6 dias e apresentou sintomas similares à argiria e suspeita de hepatotoxicidade. Embora os níveis de prata no plasma e na urina fossem altos, estes foram revertidos aos níveis normais após a remoção do curativo. ${ }^{51}$

- Em 2007, um paciente que utilizou o mesmo curativo por 28 dias apresentou no soro o valor de 56,8 $\mu \mathrm{g} \mathrm{\textrm {L } ^ { - 1 }}$ de prata. Após 3 meses do término da utilização do curativo, os valores de prata no soro foram revertidos aos níveis normais. ${ }^{52}$

- Em 2012, dois trabalhadores do sexo masculinos foram analisados após 7 anos de exposição a nanomateriais de prata em concentrações de 3,5 e 13,5 $\mathrm{ng} \mathrm{L}^{-1}$. Análises bioquímicas do sangue e as informações hematológicas foram encontradas dentro dos níveis normais. No primeiro indivíduo, foi estimada uma exposição de $0,35 \mu \mathrm{g} \mathrm{m}^{-3}$ de prata acompanhada de um nível de $0,34 \mu \mathrm{g} / \mathrm{L}$ no sangue e $0,43 \mu \mathrm{g} \mathrm{L}^{-1}$ na urina. No segundo indivíduo, a exposição estimada foi de $1,35 \mu \mathrm{g} \mathrm{m} \mathrm{m}^{-3}$ acompanhada de $0,30 \mu \mathrm{g} \mathrm{L}^{-1}$ no sangue e não detectável $\left(<0,1 \mu \mathrm{g} \mathrm{L}^{-1}\right)$ na urina. Parâmetros de química clínica e hematologia foram relatados como dentro da faixa normal. ${ }^{53}$

- Um estudo de toxicidade em 62 humanos em 2014 foi realizado com NPs de Ag produzidas por eletrólise aquosa de tamanhos variando de 5-10 $\mathrm{nm}$ (concentração estoque de $10 \mu \mathrm{g} \mathrm{mL} \mathrm{mL}^{-1}$ ) ou na faixa de $25-40 \mathrm{~nm}$ (concentração estoque de $32 \mu \mathrm{g} \mathrm{mL}^{-1}$ ). ${ }^{54} \mathrm{~A}$ ingestão diária foi de $100 \mu \mathrm{g} /$ dia para $5-10 \mathrm{~nm}$ e $480 \mu \mathrm{g} /$ dia para 25-40 nm de prata. A administração oral de prata em suspensão aquosa libera tanto prata iônica como NPs de Ag. Afirmou-se nesta pesquisa que a maioria da prata detectada em amostras de sangue do paciente foi iônica, e ausência de evidências de que NPs de Ag intactas fossem absorvidas na circulação através do trato digestivo, ou ligadas aos componentes do sangue. A conclusão final dos autores foi que a exposição oral destas NPs de Ag comerciais não produz mudanças significativas no metabolismo humano, hematológica, na urina, observações físicas ou nas imagens de morfologia. Os autores sugerem a necessidade de mais estudos para compreender melhor os riscos de toxicidade NPs de Ag por outros sistemas biológicos. 
- Munger et al. continuaram a investigar se a ingestão da prata coloidal ingerida oralmente produzia interferência na farmacocinética da enzima específica citocromo P450 em estudo cego simples, controlado, em pacientes simultaneamente com administração de sondas padronizadas da enzima P450. A ingestão oral da prata coloidal comercial produziu níveis de prata detectáveis em soro humano após 14 dias de administração, sem alteração clinicamente significativa. Foram avaliados aspectos metabólicos, hematológicos, urinários e aspectos físicos. ${ }^{55}$

\section{Casos extremos}

Uma paciente de 58 anos de idade com argiria sobreviveu após a ingestão de $1 \mathrm{~L}$ de suspensão de prata coloidal diariamente por 16 meses como remédio tradicional (valor sérico de $381 \mathrm{ng} / \mathrm{mL}$ (referência $<15 \mathrm{ng} \mathrm{mL}^{-1}$ ). ${ }^{56} \mathrm{Um}$ homem de 71 anos com câncer de próstata ingeriu prata coloidal por 4 meses, com alto teor de prata no plasma, morreu após 6 meses após a nova ingestão de prata coloidal. ${ }^{57}$ Wadhera e Fung ${ }^{58}$ demonstraram que, para argiria, uma ingestão de prata coloidal deve ser realizada três vezes por dia durante 10 meses (450 mL de uma solução de $450 \mathrm{mg} \mathrm{L}^{-1}$ ). Todos os dados mostram que os efeitos terapêutico e tóxico podem ser somente exibidos pela prata livre $\operatorname{Ag}(\mathrm{I}){ }^{59}$

Em relação à exposição por inalação, Tsai et al. ${ }^{60}$ avaliou as exposições de nanopartículas no ar associadas ao manuseio manual de nano-alumina e nano-prata em exaustores em instalações de escala de laboratório. As NPs de Ag pareciam ter um tamanho primário inferior a $100 \mathrm{~nm}$, entretanto sofreram elevada agregação em partículas de vários mm quando examinadas como um pó em massa. No entanto, para manusear $15 \mathrm{~g}$ de prata em um béquer, foram relatadas concentrações no ar com um tamanho na faixa de 100-200 nm e uma contagem de pico de 7000 partículas por $\mathrm{cm}^{3}$.

Ressalta-se que as informações referentes à ingestão de prata por humanos são amplamente restrita a casos narrativos em que humanos ingeriram determinadas quantidades de NP de Ag (provavelmente como prata coloidal), geralmente em um período longo. ${ }^{61}$ Neste caso, a doença que foi encontrada foi a chamada argiria, como mencionado anteriormente. ${ }^{62}$ Isso se deu devido ao acúmulo de diferentes graus de sulfeto de prata nas fibras elásticas. A cor da pele de pessoas com argiria varia de azulada à preta. Nesses casos, são reportados elevados níveis séricos de prata e grânulos de prata, principalmente na pele, baço, fígado e glândulas suprarrenais. ${ }^{62}$ Lyon et al. encontraram em amostras de fígado de crianças menores de seis anos de idade, a partir de amostras coletadas por autópsia, um nível significativo de prata $(15,5 \mathrm{ng} / \mathrm{g} \mathrm{m} / \mathrm{m})$, provavelmente de amálgama materna durante a gravidez e lactação. A presença de prata no fígado neonatal sugeriu que este metal atravessasse a barreira placentária em humanos. ${ }^{63}$

\section{CONCLUSÕES}

As nanopartículas de prata produzem efeitos tóxicos distintos dependendo de vários fatores discutidos nesta revisão. Resultados de estudos in vivo, tanto com camundongos quanto com humanos, demonstraram baixa toxicidade. Todavia, é evidente que existe a necessidade de estudos com maior tempo de exposição e avaliação em sistemas fisiológicos ainda não estudados. A utilização de materiais de referência (certificados) e estudos toxicológicos integrados (interlaboratoriais) são de fundamental importância para o avanço do conhecimento e regulamentação envolvendo NPs de Ag.

Ressalta-se que o mau uso das NPs de Ag poderia, em longo prazo, causar problemas para a saúde. Foi reportado um trabalho experimental que monitorou a possibilidade de NPs de Ag desenvolverem resistência a Escherichia coli (K-12MG1655), uma bactéria que, até o presente momento, não demonstra resistência à prata. Após 225 gerações de exposição ao ambiente de NPs de Ag, as populações de bactérias expostas às NPs demonstraram maiores aptidões em relação ao grupo controle para desenvolverem resistência, na presença de concentrações variáveis de NPs de Ag revestidas com citrato e de tamanho de $10 \mathrm{~nm}$. A análise genômica mostrou que as mudanças associadas à resistência bacteriana às NPs de Ag já estavam acumulando dentro das populações de tratamento pela geração 100 e, pela geração 200, três mutações tinham alcançado alta frequência nos estoques de resistência às NPs de Ag. Este estudo indicou que, apesar das reivindicações anteriores, seria difícil de conter a resistência bacteriana frente às NPs de Ag. As bactérias, contrariamente, podem facilmente evoluir resistência às NPs de $\mathrm{Ag}$, e isso ocorre por mudanças genômicas relativamente simples. Estes resultados indicam que devem ser tomados cuidados com relação ao uso de NPs de Ag como biocidas, bem como no que se refere à exposição não intencional de comunidades microbianas às NPs de Ag em produtos derivados de resíduos, ${ }^{64}$ ou quando se considerada seu uso no meio ambiente para tratar algumas doenças em animais. ${ }^{65}$

\section{AGRADECIMENTOS}

Agradecemos ao CNPq, à FAPESP (2016/10347-6) e aos centros INCT-INOMAT, NanoBioss-SisNANO, MCTI e Rede de Nanotoxicologia - Cigenanotox (CNPq/MCTI) pelo apoio.

\section{REFERÊNCIAS}

1. Selvaraj, C.; Sakkiah, S.; Tong, W.; Hong, H.; Food Chem. Toxicol. 2018, 112, 495.

2. Gerloff, K.; Landesmann, B.; Worth, A.; Munn, S.; Palosaari, T.; Whelan, M.; Comput. Toxicol. 2017, 1, 3.

3. Durán, N.; Guterres, S. S.; Alves, O. L; Em Nanotoxicology-Materials, methodologies, and assessments; Durán, N., Guterres, S. S., Alves, O. L., eds.; Springer: New York, 2014.

4. Singh, A. K.; Engineered nanoparticles: Structure, properties and mechanisms of toxicity. Academic Press: New York, 2015.

5. Grumezescu, A.; Ficai, A; Em Nanostructures for Cancer Therapy; Grumezescu, A., Ficai, A., eds.; Elsevier: London, 2017.

6. Radaic, A.; Pugliese, G. O.; Campese, G. C. V.; Pessine, F. B. T.; de Jesus, M. B.; Quim. Nova 2016, 39, 1236.

7. Metal nanoparticles in microbiology; Rai, M., Durán, N., eds.; Springer: Berlin Heidelberg, 2011.

8. Natsuki, J.; Natsuki, T.; Hashimoto, Y.; Int. J. Mat. Sci. Appl. 2015, 4, 325.

9. Abbasi, E.; Milani, M.; Aval, S. F.; Kouhi, M.; Akbarzadeh, A.; Nasrabadi, H. T.; Nikasa, P.; Joo, S. W.; Hanifehpour, Y.; Nejati-Koshki, K.; Samiei, M.; Rev. Microbiol. 2016, 42, 173.

10. Calderón-Jiménez, B.; Johnson, M. E.; Bustos, A. R. M.; Murphy, K. E.; Winchester, M. R.; Baudrit, J. R. V.; Front. Chem. 2017, 5, Art. 6.

11. Díaz-cruz, C.; Alonso, G.; Espinoza-Gómez, H.; Flores-López, L. Z.; Eur. Polym. J. 2016, 83, 265.

12. Seabra, A. B.; Manosalva, N.; De Araujo- Lima, B.; Pelegrino, M. T.; Brocchi, M., Rubilar, O.; Durán, N.; J. Phys.: Conf. Ser. 2017, 838, 12031.

13. Vazquez-muñoz, R.; Borrego, B.; Juárez-moreno, K.; García-García, M.; Mota, J. D.; Bogdanchikova, N.; Huerta-saquero, A.; Toxicol. Lett. 2017, 276, 11 .

14. Zhang, X.-F.; Liu, Z.-G.; Shen, W.; Gurunathan, S; Int. J. Mol. Sci. 2016, 17, 1534.

15. Paschoalino, M. P.; Marcone, G. P. S.; Jardim, W. J.; Quim. Nova 2010, 33,421 . 
16. De Lima, R.; Seabra, A. B.; Durán, N.; J. Appl. Toxicol. 2012, 32, 867.

17. Nogueira, P. F. M.; Paino, I. M. M.; Zucolotto, V.; Vigilância Sanitária em Debate 2013, 1, 59.

18. SCENIHR (Scientific Committee on Emerging and Newly Identified Health Risks), Nanosilver: safety, health and environmental effects and role in antimicrobial Resistance, 2013, disponível em https://ec.europa. eu/health/scientific_committees/emerging/docs/scenihr_o_039.pdf, acessado em dezembro de 2018.

19. Wang, Z.; Xia, T.; Liu, S. D.; Nanoscale 2015, 7, 7470.

20. Slavin, Y. N.; Asnis, J.; Häfeli, U. O.; Bach, H.; J. Nanobiotechnol. 2017, $15,65$.

21. Durán, N.; Seabra, A. B.; Curr. Nanosci. 2018, 14, 82

22. Mulfinger, L.; Solomon, S. D.; Bahadory, M.; Jeyarajasingam, A. V.; Rutkowsky, S. A.; Boritz, C.; J. Chem. Educ. 2007, 84, 322.

23. Zahra, A. Q.; Fraz, A.; Anwar, A.; Awais, M.; Abbas, M.; NUST Journal of Engineering Sciences 2016, 9, 1.

24. Elemike, E. E.; Onwudiwe, D. C.; Arijeh, O.; Nwankwo, H. U.; Bull. Mater. Sci. 2017, 40, 129.

25. Nakhjavani, M.; Nikkhah, V.; Sarafraz, M. M.; Shoja, S.; Sarafraz, M.; Heat Mass Transfer 2017, 53, 3201.

26. Durán, N.; Marcato, P. D.; Alves, O. L.; De Souza, G. I. H.; Esposito, E.; J. Nanobiotechnol. 2005, 3, 8 .

27. Rajput, K.; Raghuvanshi, S.; Bhatt, A.; Rai, S. K.; Agrawal, P. K.; Int. J. Curr. Microbiol. App. Sci. 2017, 6, 1513.

28. Silva, B. S. O.; Seabra, A. B.; Biointerface Res. Appl. Chem. 2016, 6, 1280 .

29. Song, J. Y.; Kim, B. S.; Bioprocess Biosyst. Eng. 2009, 32, 79.

30. Seabra A.B.; Durán N.; Metals 2015, 5, 934.

31. Lima, R.; Feitosa, L. O.; Ballottin, D.; Marcato, P. D.; Tasic, L.; Durán, N.; J. Phys.: Conf. Ser. 2013, 429, 12020.

32. Picoli, S. U.; Durán, M.; Andrade, P. F.; Durán, N.; Nanotechnol. Rev. 2016, 2, 107.

33. Melo Jr., M.A.; Santos, L. S. S.; Gonçalves, M. C.; Nogueira, S. F.; Quim. Nova 2012, 35, 1872.

34. Durán, N.; Marcato, P. D.; De Conti, R.; Alves, O. L.; Costa, F. T. M.; Brocchi, M.; J. Braz. Chem. Soc. 2010, 21, 949.

35. Durán, N.; Marcato, P. D.; Durán, M.; Yadav, A.; Gade, A.; Rai, M.; Appl. Microbiol. Biotechnol. 2011, 90,1609.

36. Durán, N.; Durán, M.; de Jesus, M. B.; Fávaro, W. J.; Nakazato, G.; Seabra, A. B.; Nanomedicine 2016, 12, 789.

37. Durán, N.; Islan, G. A.; Durán, M.; Castro, G. R.; J. Braz. Chem. Soc. 2016, 27, 1139

38. Syafiuddin, A.; Salmiati, Salim, M. R.; Kueh, A. B. H.; Hadibaratad, T.; Nur, H.; J. Chin. Chem. Soc. 2017, 64, 732.

39. Park, J-W.; Oh, J. H.; Kim, W. K.; Lee, S. K.; Bull. Environ. Contam. Toxicol. 2014, 93, 53.

40. Mallevre, F.; Alba, C.; Milne, C.; Gillespie, D.; Fernandes, T. F.; Aspray, T. J.; Nanomaterials 2016, 6, 49.

41. Van der Zande, M.; Vandebriel, R. J.; Van Doren, E.; Kramer, E.; Rivera, Z. H.; Serrano-Rojero, C. S.; Gremmer, E. R.; Mast, J.; Peters, R. J. B.; Hollman, P. C. H.; Hendriksen, P. J. M.; Marvin, H. J. P.; Peijnenburg, A. A. C. M.; Bouwmeester, H.; ACS Nano 2012, 6, 7427.
42. Das, B.; Tripathy, S.; Adhikary, J.; Chattopadhyay, S.; Mandal, D.; Dash, D. K.; Das, S.; Dey, A.; Dey, S. K.; Das, D.; Roy, S.; J. Biol. Inorg. Chem. 2017, 22, 893.

43. Durán, N.; Silveira, C. P.; Durán, M.; Martinez, D. S. T.; J. Nanobiotechnol. 2015, 13, 55.

44. Liu, W.; Wu, Y.; Wang, C.; Li, H. C.; Wang, T.; Liao, C. Y.; Cui, L.; Zhou, Q. F.; Yan, B.; Jiang, G. B.; Nanotoxicology 2010, 4, 319.

45. Bouallegui, Y.; Younes, R. B.; Turki, F.; Oueslati, R.; J. Immunotoxicol. 2017, 14, 116.

46. Tak, Y. K.; Pal, S.; Naoghare, P. K.; Rangasamy, S.; Song, J. M.; Sci. Rep. 2015, 5, 16908.

47. Kim, Y. S.; Song, M. Y.; Park, J. D.; Song, K. S. D.; Ryu, H. R.; Chung, Y. H.; Chang, H. K.; Lee, J. H.; Oh, K. H.; Kelman, B. J.; Hwang, I. K.; Yu, I. J.; Part. Fibre Toxicol. 2010, 7, 1.

48. Pinzaru, I.; Coricovac, D.; Dehelean, C.; Moacă, E. A.; Mioc, M.; Baderca, F.; Sizemore, I.; Brittle, S.; Marti, D.; Calina, C. D.; Tsatsakis, A. M.; Şoica, C.; Food Chem. Toxicol. 2018, 111, 546.

49. CONAMA - Conselho Nacional do Meio Ambiente. Resolução ${ }^{\circ}$ 357/05: Estabelece a classificação das águas doces, salobras e salinas do Território Nacional. Brasília, SEMA. 2005.

50. Rosenman, K. D.; Moss, A.; Kon, S.; J. Occup. Med. 1979, $21,430$.

51. Trop, M.; Ovak, M.; Rodl, S.; Hellbom, B.; Kroell, W.; Goessler, W.; J. Trauma 2016, 60, 648.

52. Vlachou, E.; Chipp, E.; Shale, E.; Wilson, Y. T.; Papini, R.; Moimen, N. S.; Burns 2007, 33, 979.

53. Lee, J. H., Mun, J.; Park, J. D.; Yu, I. J.; Nanotoxicology 2012, 6, 667.

54. Munger, M. A.; Radwanski, P.; Hadlock, G. C.; Stoddard, G.; Shaaban, A.; Falconer, J.; Grainger, D. W.; Deering-Rice, C. E.; Nanomedicine 2014, 10, 1 .

55. Munger, M. A.; Hadlock, G.; Stoddard, G.; Slawson, M. H.; Wilkins, D. G.; Cox, N.; Rollins, D.; Nanotoxicology 2015, 9, 474.

56. Kim, Y.; Suh, H. S.; Cham, H. J.; Kim, S. H.; Jeong, K. S.; Kim, D. H.; Am. J. Ind. Med. 2009, 52, 246.

57. Mirsattari, S. M.; Hammond, R. R.; Sharpe, M. D.; Leung, F. Y.; Young, G. B.; Neurology 2004, 62,1408.

58. Wadhera, A.; Fung, M.; Dermatol. Online J. 2005, 11, 12.

59. Eckhardt, S.; Brunetto, P. S.; Gagnon, J.; Priebe, M.; Giese, B.; Fromm, K. M.; Chem. Rev. 2013, 113, 4708.

60. Tsai, S. J. C.; Ada, E.; Isaacs, J. A.; Ellenbecker, M. J.; J. Nanopart. Res. 2009, 11, 147.

61. Chung, I. S.; Lee M. Y.; Shin, D. H.; Jung, H. R.; Int. J. Dermatol. 2010, 49, 1175.

62. Brandt, D.; Park, B.; Hoang, M.; Jacobe, H. T.; J. Am. Acad. Dermatol. 2005, 53, S105

63. Lyon, T. D. B.; Patriarca, M.; Howatson, A. G.; Fleming, P. J.; Blair, P. S.; Fell, G. S.; J. Environ. Monit. 2002, 4, 1034.

64. Graves Jr., J. L.; Tajkarimi, M.; Cunningham, Q.; Campbell, A.; Nonga, H.; Harrison, S. H.; Barrick, J. E.; Front. Genet. 2015, 6, 42.

65. Durán, N.; Durán, M.; de Souza, C. E.; J. Braz. Chem. Soc. 2017, 28, 927. 